\title{
TRPV1 as a key determinant in ciguatera and neurotoxic shellfish poisoning
}

\author{
Eva Cuypers ${ }^{\mathrm{a}}$, Angel Yanagihara ${ }^{\mathrm{b}}$, Jon D. Rainier ${ }^{\mathrm{c}}$, Jan Tytgat ${ }^{\mathrm{a}, *}$ \\ ${ }^{a}$ Laboratorium voor Toxicologie, Campus Gasthuisberg, Herestraat 49, bus 922, KULeuven, B-3000, Belgium \\ ${ }^{\mathrm{b}}$ Békésy Laboratory of Neurobiology, Pacific Biosciences Research Center, University of Hawaii at Manoa, 1993 East West Road, Honolulu, HI 96822, USA \\ ${ }^{\mathrm{c}}$ Department of Chemistry, University of Utah, 315 South 1400 East, Salt Lake City, UT 84112, USA
}

Received 3 July 2007

Available online 16 July 2007

\begin{abstract}
Ciguatera fish poisoning and neurotoxic shellish poisoning are distinct clinical entities characterized by gastrointestinal and neurological disturbances, following the consumption of certain reef fish and shellfish containing toxic polyether compounds sporadically present in certain toxic marine dinoflagellates. The biotransformation and bioaccumulation of gambierol and brevetoxin, and their congeners, are believed to be involved in the pathogenesis of these "food-chain diseases", for which no effective treatments are available. Here, we describe for the first time the potent effect of gambierol and brevetoxin on TRPV1 channels, a key player in thermal and pain sensation. Our findings may lead to promising new therapeutic interventions.
\end{abstract}

(c) 2007 Elsevier Inc. All rights reserved.

Keywords: TRPV1; Gambierol; Brevetoxin; Ciguatera fish poisoning; Neurotoxic shellfish poisoning

Characterized by gastrointestinal (nausea, vomiting, diarrhoea, and abdominal cramping) and neurological (paraesthesias, temperature reversal, and cardiac arrhythmias) signs and symptoms, ciguatera fish poisoning (CFP) and neurotoxic shellfish poisoning (NSP) are acute intoxications resulting from the consumption of tropical reef fishes and shellfish [1]. Neurological disturbances typically include tingling of the lips, hands, and feet, unusual temperature perception [2], burning mouth syndrome [3], numbness in the extremities, and pain. Several polyether toxins, such as gambierol and brevetoxin, are believed to underlie these clinical findings but very little is known about their molecular targets and their mechanisms of action. Since TRPV1, a non-selective cation channel expressed in nociceptive neurons, is known to be involved in temperature and burning-pain sensation [4,5], we examined whether TRPV1 is involved in these typical neurological disturbances.

\footnotetext{
${ }^{*}$ Corresponding author. Fax: +3216323405 .

E-mail address: Jan.Tytgat@pharm.kuleuven.be (J. Tytgat).
}

\section{Materials and methods}

Compound synthesis and solution preparation. Gambierol was synthesized as described previously [6-8], dissolved in DMSO, and diluted in ND96. Brevetoxin (PbTx-3 from Latoxan) is dissolved in ND96. Linoleoyl ethanolamide (NAE 18:2 from Cayman Chemical) was diluted in ND96 solution. The total DMSO concentration was maximum $0.5 \%$.

RNA preparation and electrophysiological recordings. cRNA transcripts were synthesized from XbaI-linearized VR1 cDNA templates using T7 RNA polymerase (Ambion). Oocytes, harvested from anaesthetized female Xenopus laevis frogs as previously described [9], were injected with $0.5-5 \mathrm{ng}$ TRPV1 cRNA. Two to seven days after injection, two-electrode voltage-clamp recording was performed. Currents were measured in ND96 solution using a protocol of $-90 \mathrm{mV}$ during $400 \mathrm{~s}$. Current-voltage (I-V) curves were obtained using a series of $400 \mathrm{~ms}$ step pulses from -90 to $+90 \mathrm{mV}$. The recording chamber was perfused at a rate of $2 \mathrm{~mL} \mathrm{~min}^{-1}$ with a ND-96 solution containing (in mM) $96 \mathrm{NaCl}, 2 \mathrm{KCl}, 1.8 \mathrm{CaCl}_{2}, 1 \mathrm{MgCl}_{2}$, and 5 HEPES, pH 7.4 Temperature of the perfusate was kept at $22{ }^{\circ} \mathrm{C}$ and controlled using a SC-20 dual in-line heater/cooler (Warner Instruments) and pH was kept at 7.4 unless otherwise described. As previously described [10], capsaicin $(2 \mu \mathrm{M})$ was used as an agonist and capsazepine $(10 \mu \mathrm{M})$ as an antagonist of TRPV1. Capsaicin and capsazepine were purchased from Sigma, and anandamide from Tocris. To obtain $\mathrm{EC}_{50}$ values, the toxininduced total current was normalized to the capsaicin current $(=100 \%)$, 
A

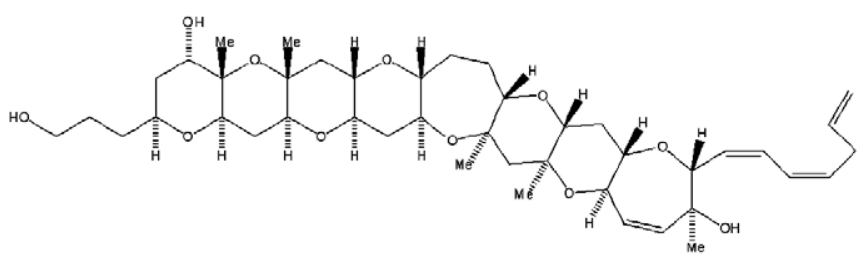

Gambierol
B

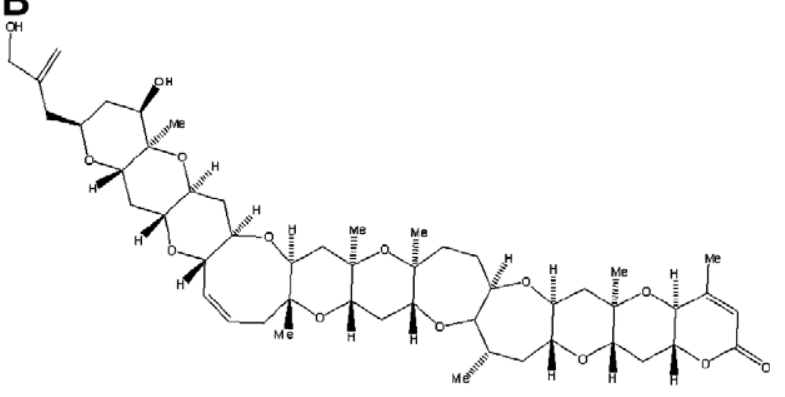

Brevetoxin

Fig. 1. Structure of gambierol (A) and brevetoxin (B).

A

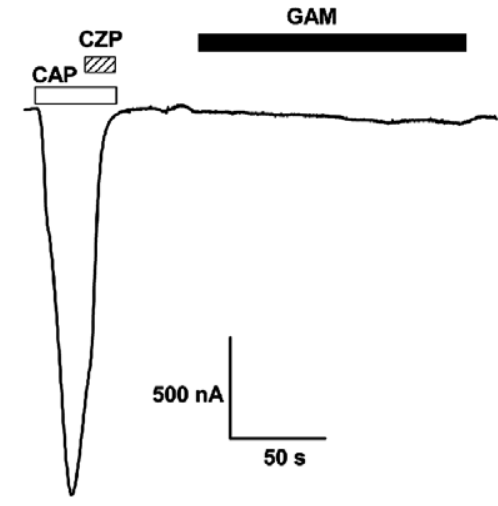

C

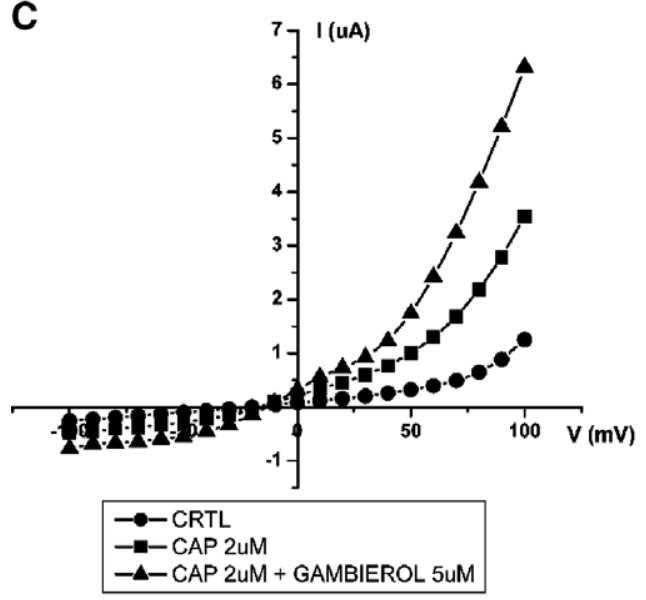

B

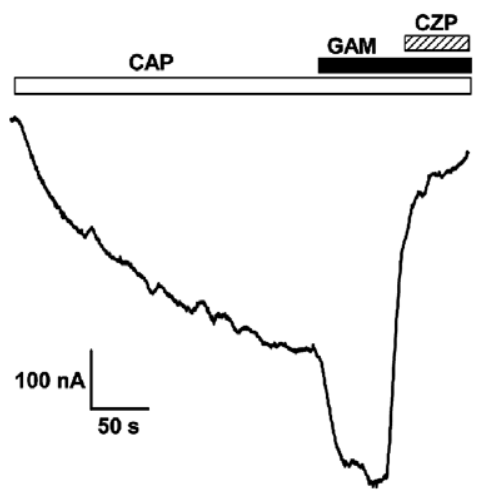

D

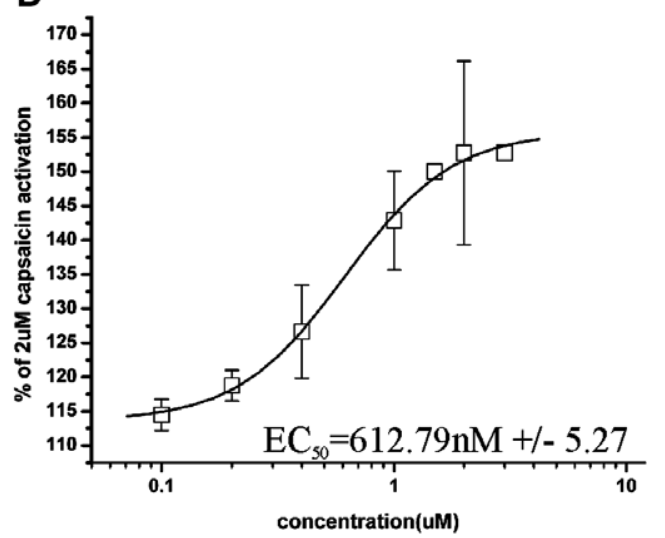

E

NAE 18:2

GAM

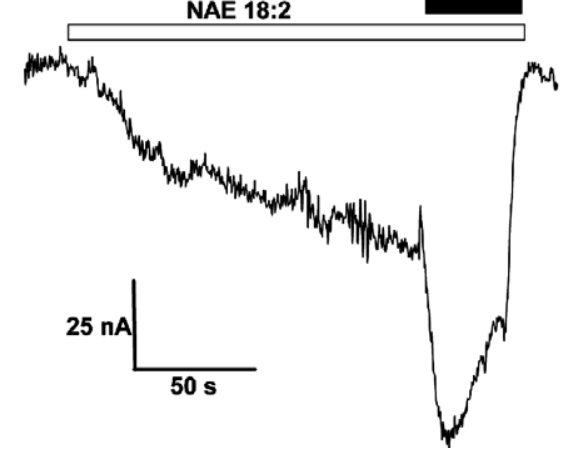

Fig. 2. Electrophysiological tests of gambierol alone (A) and in the presence of capsaicin (B). I-V curve and dose-response of the allosteric effect are shown in, respectively, (C) and (D). (E) Current showing the allosteric effect of gambierol with linoleoyl ethanolamide (NAE 18:2) as endogenous activator. 
plotted against the concentration of toxin used, and a fit with the Hill equation yielded the $\mathrm{EC}_{50}$.

\section{Results}

Administration of $10 \mu \mathrm{M}$ gambierol (Fig. 1A) showed no visible effect on TRPV1 channels (Fig. 2A). In contrast, when gambierol $(10 \mu \mathrm{M})$ was applied together with capsaicin, a clear allosteric effect with an $\mathrm{EC}_{50}$ of $613 \mathrm{nM}$ (Fig. 2B and D) was found. I-V curve is shown in Fig. 2C. No allosteric effect was visible when the same testing method was used with a ND96 solution of pH 5.4 as an activator of TRPV1 channels instead of capsaicin (data not shown). These results indicate that the acting site of gambierol is most likely related to the intracellular binding site of capsaicin, in contrast with the extracellular binding site of protons. Fig. 2E shows the same allosteric effect when linoleoyl ethanolamide was used as endogenous activator instead of capsaicin.

The polycyclic ether toxin involved in NPS is brevetoxin (Fig. 1B). In order to see whether the described effects are a hallmark for this group of toxins, brevetoxin ( $\mathrm{PbTx}-3$ from Latoxan) was also tested on TRPV1. Fig. 3A shows there is no effect of brevetoxin $(2 \mu \mathrm{M})$ alone on TRPV1, although an allosteric effect, with an $\mathrm{EC}_{50}$ of $352 \mathrm{nM}$, was visible when capsaicin $(2 \mu \mathrm{M})$ was added together with brevetoxin
(Fig. 3B and D). Fig. 3C shows the $\mathrm{I}-\mathrm{V}$ curve. Using an acidic ND96 solution ( $\mathrm{pH} 5.4$ ) instead of capsaicin as an activator did not give an allosteric effect (data not shown).

\section{Discussion}

The described effects seem to be general for the group of polycyclic ether toxins inducing CFP and NSP. Typical neurological symptoms, like burning mouth syndrome and unusual temperature sensation [2,3], can be explained as follows: endogenous activators like anandamide [11] or unsaturated fatty acids (e.g., linoleoyl ethanolamide) [12] may give a constant small activation of TRPV1 channels. When contaminated fish is eaten, polycyclic ether toxins come into contact with the endogenous activated TRPV1 channels and the described allosteric effect leads to an increased inward current of cations. This inward current is well known to be associated with a typical burning-pain sensation $[10,13]$ and to play a key role in temperature sensation [5].

An interesting mechanistic convergence is our finding of allosteric effects on TRPV1 by compounds isolated from cnidarian sources [14]. It is intriguing to consider this in the context of a case report of CFP associated with jellyfish (cnidaria) ingestion [15]. Further, recent and ongoing studies by us demonstrate the presence of a diverse suite of bio-
A

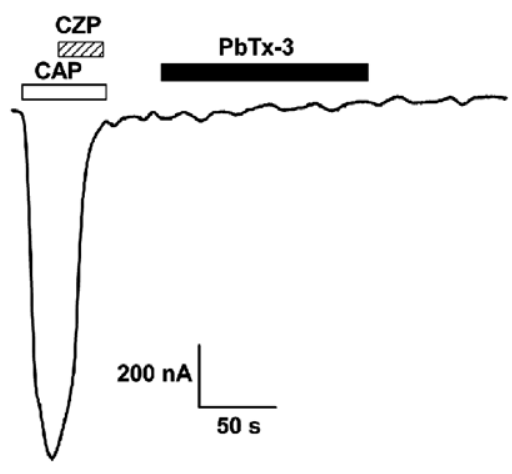

C

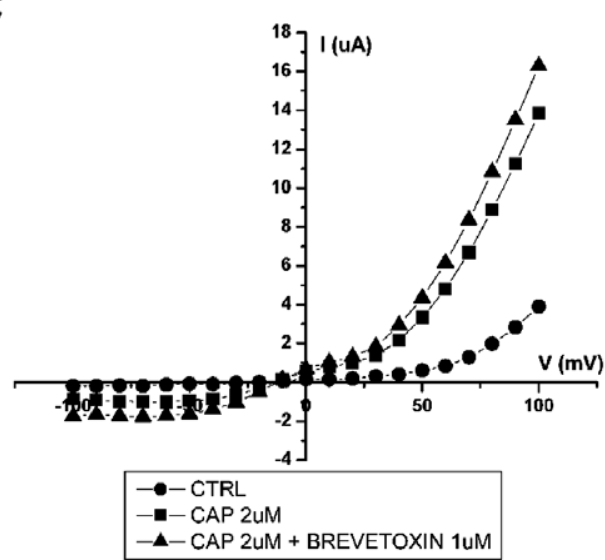

B

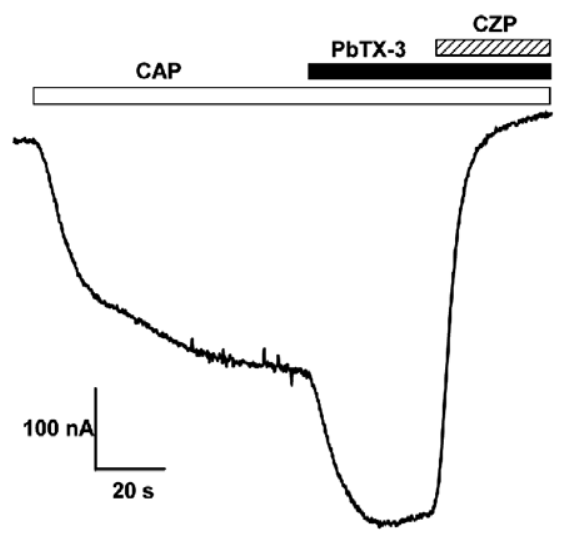

D

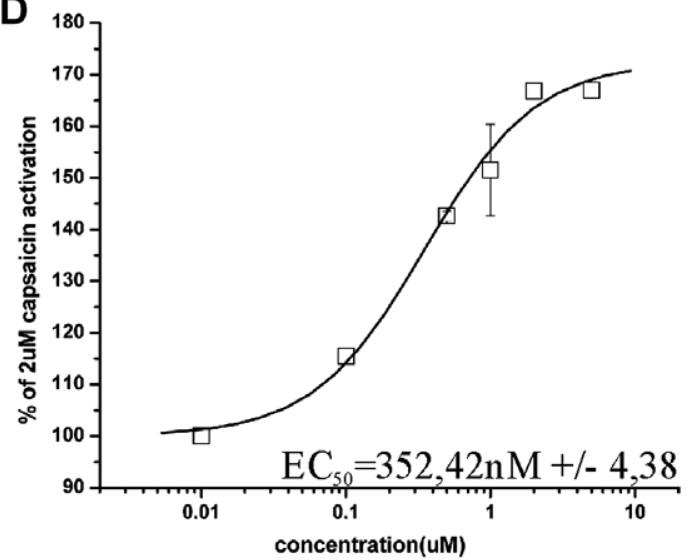

Fig. 3. Electrophysiological tests of brevetoxin alone (A) and in the presence of capsaicin (B). I-V curve and dose-response of the allosteric effect are shown in, respectively, (C) and (D). 
active lipidic compounds amongst the active cocktail of substances in cnidaria venoms.

In conclusion, the elucidation that polycyclic ether toxins activate TRPV1 is novel and may lead to the development of effective therapies. Work to further elucidate the mechanism by which the toxins activate TRPV1 is ongoing.

\section{Acknowledgments}

We thank Dr. David Julius for providing the rat TRPV1 clone and Sarah Debaveye for support in molecular biology work. This work was supported by the following Grants: OT-05-64 (K.U.Leuven), G.0330.06 (F.W.O.-Vlaanderen), and P6/31 (Interuniversity attraction Poles Programme-Belgian State-Belgian Science Policy), GM 56677 (NIGMS, J.D.R.). Dr. Yanagihara's work was supported by 20061497, Hawaii Community Foundation, Honolulu, Hawaii.

\section{References}

[1] R.J. Lewis, Ciguatera: Australian perspectives on a global problem, Toxicon 48 (2006) 799-809.

[2] J. Cameron, M.F. Capra, The basis of the paradoxical disturbance of temperature perception in ciguatera poisoning, J. Toxicol. Clin. Toxicol. 31 (1993) 571-579.

[3] G.M. Heir, Ciguatera neurotoxin poisoning mimicking burning mouth syndrome, Quintessence Int. 36 (2005) 547-550.
[4] D.E. Clapham, TRP channels as cellular sensors, Nature 426 (2003) $517-524$.

[5] A. Patapoutian, TRP channels and thermosensation, Chem. Senses 30 (Suppl. 1) (2005) i193-i194.

[6] H.W. Johnson, U. Majumder, J.D. Rainier, The total synthesis of gambierol, J. Am. Chem. Soc. 127 (2005) 848-849.

[7] H.W. Johnson, U. Majumder, J.D. Rainier, Total synthesis of gambierol: subunit coupling and completion, Chemistry 12 (2006) 1747-1753.

[8] U. Majumder, J.M. Cox, H.W. Johnson, J.D. Rainier, Total synthesis of gambierol: the generation of the $\mathrm{A}-\mathrm{C}$ and $\mathrm{F}-\mathrm{H}$ subunits by using a C-glycoside centered strategy, Chemistry 12 (2006) 1736-1746.

[9] E.R. Liman, J. Tytgat, P. Hess, Subunit stoichiometry of a mammalian $\mathrm{K}+$ channel determined by construction of multimeric cDNAs, Neuron 9 (1992) 861-871.

[10] M.J. Caterina, M.A. Schumacher, M. Tominaga, T.A. Rosen, J.D. Levine, D. Julius, The capsaicin receptor: a heat-activated ion channel in the pain pathway, Nature 389 (1997) 816-824.

[11] D. Smart, J.C. Jerman, Anandamide: an endogenous activator of the vanilloid receptor, Trends Pharmacol. Sci. 21 (2000) 134.

[12] P. Movahed, B.A. Jonsson, B. Birnir, J.A. Wingstrand, T.D. Jorgensen, A. Ermund, O. Sterner, P.M. Zygmunt, E.D. Hogestatt, Endogenous unsaturated $\mathrm{C} 18 \mathrm{~N}$-acylethanolamines are vanilloid receptor (TRPV1) agonists, J. Biol. Chem. 280 (2005) 38496-38504.

[13] M. Numazaki, M. Tominaga, Nociception and TRP channels, Curr. Drug Targets CNS Neurol. Disord. 3 (2004) 479-485.

[14] E. Cuypers, A. Yanagihara, E. Karlsson, J. Tytgat, Jellyfish and other cnidarian envenomations cause pain by affecting TRPV1 channels, FEBS Lett. 580 (2006) 5728-5732.

[15] B.A. Zlotnick, S. Hintz, D.L. Park, P.S. Auerbach, Ciguatera poisoning after ingestion of imported jellyfish: diagnostic application of serum immunoassay, Wilderness Environ. Med. 6 (1995) 288-294. 